\title{
KDEL Receptor Expression Is Not Coordinatedly Up-regulated with ER Stress-Induced Reticuloplasmin Expression in HeLa Cells
}

\author{
David H. Llewellyn, ${ }^{1}$ H. Llewelyn Roderick, and Sally Rose \\ Department of Medical Biochemistry, University of Wales College of Medicine, Heath Park, Cardiff CF 4 4XN, Wales
}

Received October 3, 1997

\begin{abstract}
Perturbation of the normal functioning of the endoplasmic reticulum (ER) increases the expression of lumenal proteins, such as grp78, and calreticulin. These proteins are retained within the compartment by a salvage mechanism involving the recognition of a C-terminal tetra-peptide sequence by the KDEL receptor. We have investigated whether disrupting normal ER function concomitantly increases the expression of the mRNAs encoding the two mammalian isoforms of the receptor, erd2.1 and erd2.2. Inhibition of $\mathrm{N}$-linked glycosylation of proteins by tunicamycin had no effect upon the levels of the single mRNA species recognised by the erd2.1 probe, or the multiple transcripts detected with the erd2.2 cDNA probe. $E R \mathrm{Ca}^{2+}$ store depletion by thapsigargin did not increase erd2.1 mRNA, but actually caused a decrease in erd2.2 mRNA. Both thapsigargin, and tunicamycin, increased calreticulin secretion from the cells, although this might be due to more than simply saturation of KDEL receptor binding. 1997 Academic Press
\end{abstract}

The endoplasmic reticulum (ER) is the major calcium store in non-muscle cells and, also, the site where proteins destined for secretion, or insertion in the plasma membrane, are initially folded and assembled. Amongst the proteins that facilitate these processes are a group of calcium-binding proteins located within thelumen of theER called the reticuloplasmins, including BiP/grp78, endoplasmin/grp94, protein disulphide isomerase (PDI), and cal reticulin [1].

These proteins are targeted to the $E R$ by virtue of an $\mathrm{N}$-terminal hydrophobic signal sequence that is cleaved following translocation into the ER lumen. Once in the lumen, their presence is maintained by a retention and

\footnotetext{
${ }^{1}$ To whom correspondence should be sent. E-mail: Ilewellyndh@ cardiff.ac.uk. Fax: 441222766276.

Abbreviations used: $E R$, endoplasmic reticulum; KDEL-R, KDEL receptor; grp, glucose-response protein; PCR, polymerase chain reaction.
}

retrieval mechanism dependent upon receptor recognition of a tetra-peptide sorting signal at the C-terminus [2]. In yeast, this tetra-peptide sequence found in the equivalent proteins is HDEL (His-Asp-Glu-Leu) [3], but in higher eukaryotes, it is usually KDEL (Lys-AspGlu-Leu) [4], although variants such as HNEL also exist [5]. In Saccharomyces cerevisiae, the HDEL receptor is encoded by the erd2 gene, which is essential for growth [6]. Its product, erd2p is an integral membrane protein predicted to have seven trans-membrane spanning domains [7]. The mammalian KDEL receptor appears to be a similar type of protein with isoforms encoded by two mRNAs, erd2.1, and erd2.2 [8-10].

In response to perturbation of normal ER function, such as chronic depletion of the ER Ca ${ }^{2+}$ store by calcium ionophores or thapsigargin, or inhibition of glycosylation by tunicamycin, expression of the genes encoding KDEL-containing proteins is induced in a wide variety of cells [11-14]. There have been conflicting reports about whether such reticuloplasmin overexpression leads to the secretion of these proteins from cells. In 3 T 3 and some other murine cells exposed to the calcium ionophore A23187 for 4-8 hours, their secretion was reported [15], whilst other studies have shown that similar treatment of 3T3 cells, as well as HepG2 cells, does not lead to their loss [16]. This might indicate the existence of a preventative mechanism. One possibility is that there is a concomitant increase in KDEL receptor expression in response to ER stress. Indirect evidence for such a proposal comes from a number of observations.

Overexpression of the erd2p protein in yeast resulted in more efficient retrieval of HDEL proteins, whilst, conversely, reduced expression of erd2p led to their secretion [17]. In human glioblastoma U 87 cells, overexpression of either erd2.1 or erd2.2 proteins increased the retention of KDEL-and HNEL-containing proteins, and overexpression of human growth hormone, to which either KDEL or HNEL had been appended at the C-terminus, caused the secretion of endogenous re- 
ceptor-associated protein (RAP), a KDEL-containing $E R$ resident protein, as a result of receptor saturation [18]. Furthermore, in Arabidopsis, tunicamycin induced expression of aerd2 mRNA that encodes the plant cell homologue of the KDEL receptor [19].

Therefore, in this study, we investigated the effect of perturbation of ER function upon expression of the two human KDEL receptors, and the secretion of calreticulin, in HeLa cells.

\section{MATERIALS AND METHODS}

Thapsigargin and tunicamycin were obtained from Calbiochem (Nottingham, England). All other chemicals were the highest grade available and obtained either from Sigma (Poole, England), or Fisons (Loughborough, England).

Ultraspec reagent was obtained from AMS (Witney, England). Restriction and modifying enzymes were bought from Gibco-Life Technologies (Paisley, Scotland), or Promega (Southampton, England). Rapid-Hyb hybridisation buffer, RediPrime labelling kit and ${ }^{32} \mathrm{P} \alpha$ dCTP (Redivue AA0005) came from Amersham International (Amersham, Bucks, England). Oligonucleotides were synthesised by the UWCM molecular biology services facility.

Cells and culture conditions. Tissue-culture media and supplements were obtained from Sigma, or Gibco-Life technologies Adherent HeLa OHIO cells were maintained in Dulbecco's modified Eagle medium (DMEM) $+10 \%$ fetal calf serum (FCS). For treatment with thapsigargin, or tunicamycin, cells were grown to $75-80 \%$ confluence, and agents added in DMEM + 10\% FCS supplemented to which $1 \mathrm{mM}$ EGTA (pH 7.6) had been added.

Generation of cDNA probes. The probes for calreticulin, grp78, and GAPDH, were obtained as previously described [14]. CDNA fragments for the human KDEL 1 (erd2.1) and KDEL 2 (erd2.2) receptors were generated by reverse-transcription (RT)-PCR using total RNA isolated from HeLa cells. Primers were derived from the published sequences [8-10].

erd2.1 forward primer, 5' GGTCCCTCTTCCGTCCCCCCA 3', reverseprimer, 5' CCACTGTCCCCCTGAAACCCG 3'.

erd2.2 forward primer, 5' CTACCTTGCCTGCTCCTATG 3' reverse primer， 5'GAAAGGACAGATGCTGGTGA 3'

Amplification products, erd2.1 - 475bp, erd2.2 - 903bp, were verified as the correct products by sequencing and comparison with the known sequences. They were used as probes in RNA blot hybridisations either directly following clean-up (Qiagen PCR preps), or cloned into the pGEM-T vector (Promega) from which appropriate fragments were excised.

Northern bl ot hybridisation. $5 \mu \mathrm{g}$ of total RNA, isolated using UItraspec, were electrophoresed through $1 \%$ agarose gels containing $0.66 \mathrm{M}$ formaldehyde, transferred by capillary action onto Zeta Probe charged nylon membranes (Bio-Rad) and cross-linked with UV light using an Amersham cross-linker. Hybridisations were performed in Rapid-Hyb buffer for 1 hour at $65^{\circ} \mathrm{C}$ with ${ }^{32} \mathrm{P}$ randomly-labelled probes. Following hybridisation, membranes were extensively washed with $0.1 \times \mathrm{SSC}, 0.1 \% \mathrm{SDS}$ at $65^{\circ} \mathrm{C}$ and exposed to X-ray film or analysed using a BioRad GS Phosphorimager. Where film was used, the relative mRNA levels were quantified by densitometry (BioRad GS-670 densitometer). Blots were stripped and re-probed with a CDNA encoding glyceraldehyde 3' phosphate dehydrogenase (GAPDH) (Ambion, Texas, USA) to ensure equivalent loading of the RNA samples, and values for calreticulin, grp78 and KDEL receptor
mRNAs were normalised to those for GAPDH. Equivalent loading was also assessed by visualisation of ethidium bromide stained $28 \mathrm{~S}$ and $18 \mathrm{~S}$ rRNA bands.

Metabolic labelling of proteins and immunoprecipitation of cal reticulin. Cells were seeded in $60 \mathrm{~mm}$ dishes and grown to $75 \%$ confluence. They were washed twice with PBS and then incubated in serum-free medium minus methionine for 1 hour at $37^{\circ} \mathrm{C}$. The culture medium was replaced with fresh DMEM $+10 \%$ FCS containing ${ }^{35} \mathrm{~S}$ L-methionine $(70 \%) /^{35} \mathrm{~S}$ L-cysteine (30\%) (Amersham Promix Cat. No. SJ Q 007), and the cells incubated for 5 hours at $37^{\circ} \mathrm{C}$ after which they were placed in normal medium containing either thapsigargin $(300 \mathrm{nM})$, tunicamycin $(10 \mu \mathrm{g} / \mathrm{ml})$, or no agent at all. The incubation continued for a further 21 hours before calreticulin was immunoprecipitated from the concentrated medium using an anti-calreticulin antibody raised against the whole human protein [20].

\section{RESULTS}

HeLa cells were exposed to thapsigargin (300nM), or tunicamycin $(10 \mu \mathrm{g} / \mathrm{ml})$, for up to 21 hours in order to induce the overexpression of resident ER proteins. Confirmation that these agents elicited an ER-stress response was carried out by N orthern analysis using calreticulin and grp78 cDNA probes. As shown in figure $1 A$, upon exposure to thapsigargin, the calreticulin mRNA levels had increased by 3 -fold by 8 hours, and 5.1-fold after 21 hours. With tunicamycin, the respective increases were 4.2-fold and 6.5 fold (figure 1B). Even greater induction was seen for grp78 mRNA in response to both agents (data not shown), in concurrence with our previous reports [14]. No changes were observed in the levels of mRNA encoding the housekeeping enzyme, glyceraldehyde $3^{\prime}$ phosphate dehydrogenase (GAPDH) with either thapsigargin or tunicamycin (figures $1 A$ and $B$ ). We also investigated whether there had been an increase in calreticulin protein. Using an anti-cal reticulin antibody, immuno-blotting of cell lysates showed that 21 hours continuous treatment with thapsigargin increased the amount of cal reticulin protein 1.9-fold, whilst prolonged exposure to tunicamycin over the same time period induced a 2.2-fold increase in its levels (figure 2).

To see whether there had been a concomitant increase in erd2.1 and erd2.2 mRNAs, the Northern blots were stripped and probed sequentially with the appropriate cDNA fragments. Hybridisation with the erd2.1 probe detected a single mRNA species of approximately $2.5 \mathrm{~kb}$ whose levels remained unchanged throughout the 21 hour exposure of the cells to either thapsigargin or tunicamycin (figure 1). The erd2.2 cDNA probe detected five bands in all the experiments performed despite the fact that the hybridisations and subsequent washes were performed at very high stringencies. The major transcript was at approximately $3.2 \mathrm{~kb}$, with a less intense band at $2.3 \mathrm{~kb}$, a very faint band at $1.7 \mathrm{~kb}$, and 2 bands at about $1.3-$ $1.4 \mathrm{~kb}$ (figure 1). No alterations were observed in the levels of any of these transcripts in response to tunicamycin (figure 1B). However, a drop in the levels of 


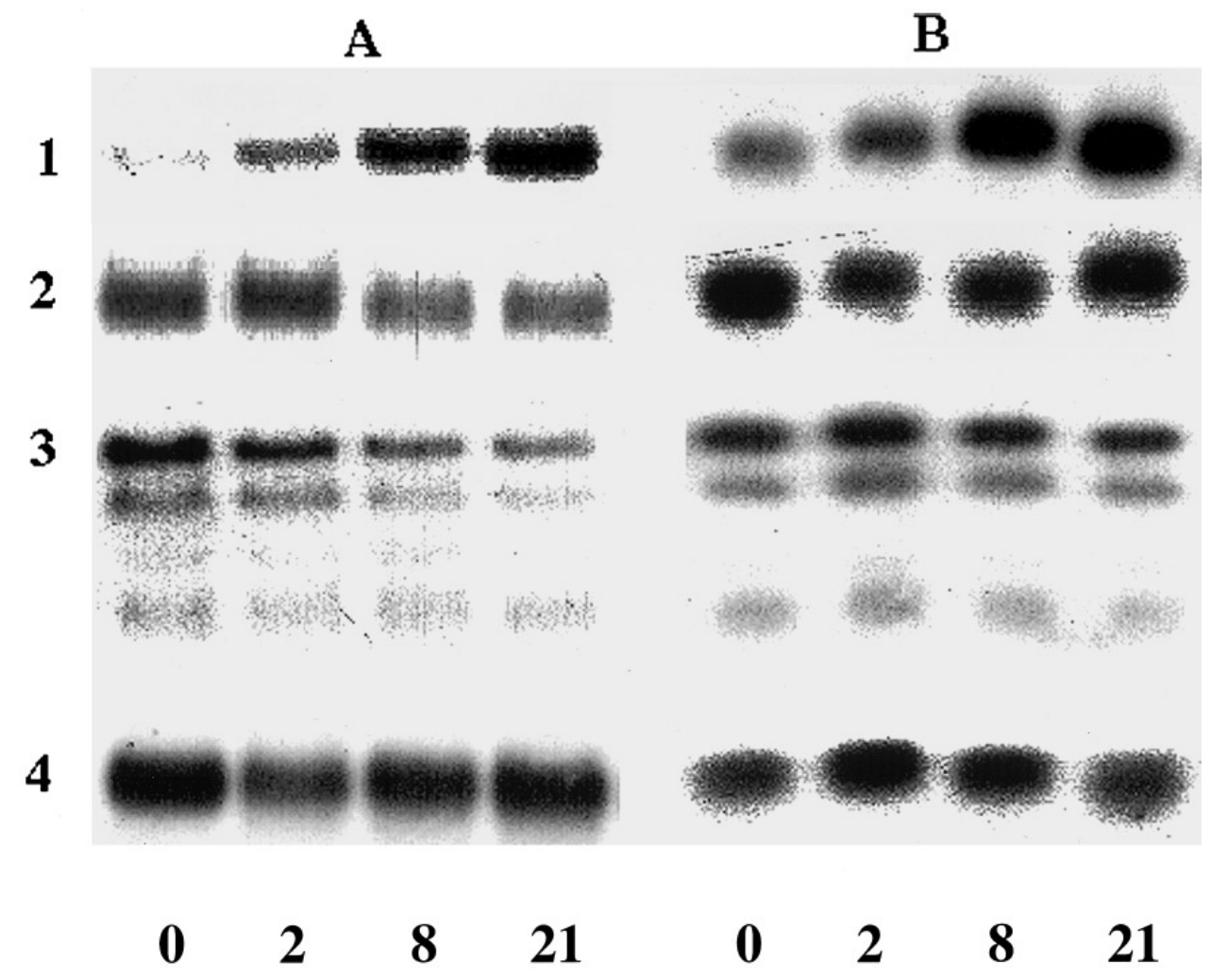

FIG. 1. Effect of ER stress upon calreticulin, erd2.1, erd2.2, and GAPDH mRNAs. Cells were exposed to either thapsigargin (300nM) (PANEL A), or, tunicamycin (10 $\mu \mathrm{g} / \mathrm{ml})$ (PANEL B), for 2, 8, and 21 hours as indicated below the lanes. Northern analysis was performed on extracted total RNA $(5 \mu \mathrm{g})$ using ${ }^{32} \mathrm{P}$ randomly labelled cDNA probes, and the results analysed using a BioRad GS Phosphorimager and Phosphor Analyst software. The results shown are typical of those seen in four independent experiments. KEY: 1:- calreticulin mRNA (1.95kb). 2:- erd2.1 mRNA (2.5kb). 3:- erd2.2 mRNA (Bands at 3.2kb, 2.3kb, 1.7kb, and 1.3-1.4kb). 4:- GAPDH mRNA (1.4kb).

all these mRNAs was observed in response to exposure to thapsigargin. In the case of the $3.2 \mathrm{~kb}$ major band, its levels had decreased by $22 \%$ and $48 \%$ at 6 and 21 hours respectively (figure $1 \mathrm{~A}$ ).

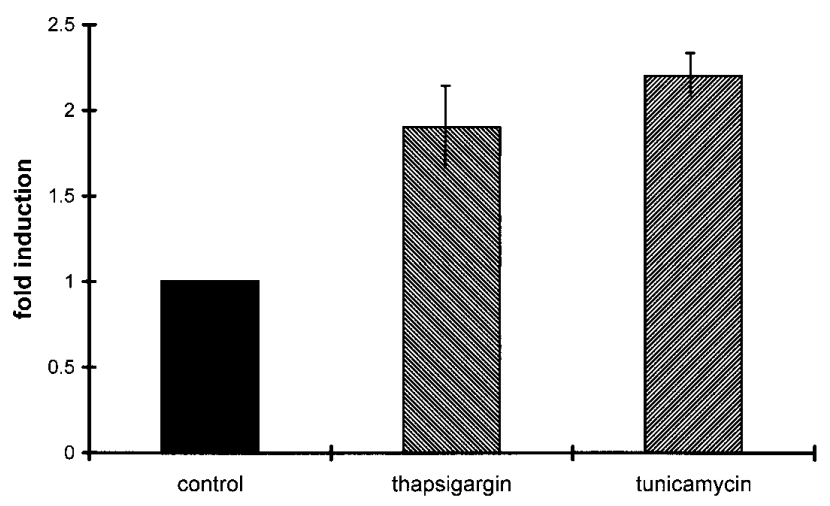

FIG. 2. Effect of thapsigargin and tunicamycin treatment upon levels of cal reticulin protein. Cells were exposed to either thapsigargin $(300 \mathrm{nM})$ or, tunicamycin $(10 \mu \mathrm{g} / \mathrm{ml})$, for 21 hours. Immuno-blotting of cell lysates was performed using an anti-cal reticulin antibody which detected a single band migrating at a mobility of approx. 60kD. Results shown are the mean inductions of four independent experiments \pm SEM.
These results led us to investigate whether the ER stress had triggered an increase in the secretion of calreticulin from the cells. This was done by monitoring the effects of prolonged treatment with thapsigargin, or tunicamycin, upon cells that had been previously incubated in normal medium containing ${ }^{35} \mathrm{~S}$ methionine/cysteine for 5 hours to produce a pool of labelled correctly-folded proteins. In control cells, not exposed to either agent, very little cal reticul in was secreted into the medium. The amount of calreticulin protein in the chase medium increased, relative to that observed with control cells, by 2.7 fold with tunicamycin, and 2 fold with thapsigargin (Table 1).

\section{DISCUSSION}

These data demonstrate conclusively that, despite increasing expression of proteins normally retained in the ER by their interaction with the KDE L receptor, perturbation of ER function does not concomitantly induce the mRNAs encoding isoforms of that receptor. These results contrast with the induction of aerd2 mRNA in ARabidopsis by tunicamycin [19]. Levels of the single $2.5 \mathrm{~kb}$ band recognised by the 
TABLE 1

Stress-Induced Calreticulin Secretion

Relative calreticulin secretion
Cell treatment

Control

+ thapsigargin

+ tunicamycin
1.00

$1.99 \pm 0.14$

$2.68 \pm 0.32$
Densitometric analysis of ${ }^{35} \mathrm{~S}$ labelled calreticulin immunoprecipitated from culture medium following stress induction by either thapsigargin $(300 \mathrm{nM})$, or, tunicamycin $(10 \mu \mathrm{g} / \mathrm{ml})$ for 18 hours. The data are the means of 3 reproducible experiments performed in duplicate \pm SEM.

erd2.1 probe were unaffected by treatment with either tunicamycin, or thapsigargin. Somewhat unexpected were the findings that, despite the use of high stringency hybridisations and washes, the erd2.2 probe detected multiple mRNA species whose levels remained constant upon exposure of the cells to tunicamycin, but dropped as a consequence of thapsigargin treatment. This probe was derived from the $3^{\prime}$ untranslated region of the published erd2.2 mRNA sequence. Whilst it is possible that there are related mRN As, in addition to erd2.1, perhaps a more plausible explanation is al ternative splicing of the erd2.2 pre-mRNA producing several mRNAs with the same $3^{\prime}$ termini. To our knowledge, neither of the two KDEL-receptor genes has yet been fully characterised, and so this proposal remains speculative at this time. The lack of induction seen with the KDELreceptor genes suggests that it is unlikely, however, that their $5^{\prime}$ regulatory regions are similar to those of calreticulin and the other reticuloplasmins $[14,21]$.

The simplest interpretation of our data showing enhanced calreticulin secretion is that it occurs as a result of saturation of KDEL receptor binding, due to the inability of HeLa cells to induce receptor expression in response to ER stress. Corroborating evidence for this proposition comes from the saturation of KDEL-receptor by overexpression of KDEL- and HNEL-tagged growth hormone in U87 cells leading to the secretion of endogenous RAP [18]. Moreover, the secretion of grp94/endoplasmin, grp78/BiP, and PDI in a range of murine cell lines as a result of disruption of ER lumenal $\mathrm{Ca}^{2+}$ has been reported previously [15]. However, in that study, unlike our data, tunicamycin failed to enhance the secretion of KDE Lcontaining proteins. The reasons for the difference between the data are not clear, but the implication is that the failure to retain these proteins within the ER may not be entirely a consequence of receptor saturation. Further evidence for such a conclusion comes from the observation that exogenous overexpression of calreticulin had no effect upon its distri- bution within cells, or its secretion [20, 22]. Conversely, the removal of its KDEL sequence did not cause a huge increase in its secretion [22]. Clearly, the mechanisms for retention of ER proteins in mammalian cells are complex and entail more than simple salvage by the KDEL receptor, e.g. maintenance of the intralumenal $\mathrm{Ca}^{2+}$ is essential for the correct processing of proteins within the ER and is likely to be involved in the retention mechanism [16, 23]. In yeast, there is a second retention system involving the ER-nuclear trans-membrane kinase Irelp, a key protein involved in ER-nuclear signalling [24]. No homol ogue of this kinase has been reported in mammalian cells, where the signalling pathways responsible for transducing information from the lumen of the $E R$ to the nucleus remain, as yet, poorly understood. F urther studies on these pathways should elucidate the processes by which the expression of ER lumenal proteins is regulated in mammalian cells, and may also shed further light on the mechanisms of their $E R$ retention.

\section{REFERENCES}

1. Macer, D. R. J ., and Koch, G. L. E. (1988) J ournal of Cell Science 91, $61-70$.

2. Pelham, H. R. B. (1995) Current Opinion in Cell Biology 7, 530535.

3. Pelham, H.R.B., Hardwick, K.G., and Lewis, M.J. (1988) EMBO J ournal 7, 1757- 1762.

4. Munro, S., and Pelham, H. R. B. (1987) Cell 48, 899- 907.

5. Bu, G. J ., Geuze, H. J ., Strous, G. J ., and Schwartz, A. L. (1995) EMBO J ournal 14, 2269- 2280.

6. Townsley, F. M., Frigerio, G., and Pelham, H. R. B. (1994) J ournal of Cell Biology 127, 21-28.

7. Townsley, F.M., Wilson, D.W., and Pelham, H.R.B. (1993) EMBO J ournal 12, 2821-2829.

8. Lewis, M. J ., and Pelham, H. R. B. (1990) Nature 348, 162- 163.

9. Lewis, M. J ., and Pelham, H. R. B. (1992) J ournal of Molecular Biology 226, 913-916.

10. Hsu, V. W., Shah, N., and Klausner, R. D. (1992) Cell 69, 625635.

11. Lee, A. S. (1987) TIBS 12, 20-23.

12. Macer, D. R. J ., and Koch, G. L. E. (1988) J ournal of Cell Science 91, 61- 70 .

13. Dorner, A. J ., Wasley, L. C., Raney, P., Haugejorden, S., Green, M., and Kaufman, R. J . (1990) J ournal of Biological Chemistry 265, 22029- 22034.

14. Llewellyn, D. H., Kendall, J.M., Sheikh, F. N., and Campbell, A. K. (1996) Biochemical J ournal 318, 555- 560.

15. Booth, C., and Koch, G. L. E. (1989) Cell 59, 729- 737.

16. Lodish, H. F., and Kong, N. (1990) J ournal of Biological Chemistry 265, 10893-10899.

17. Semenza, J . C., Hardwick, K. G., Dean, N., and Pelham, H. R. B. (1990) Cell 61, 1349-1357.

18. Bu, G. J ., Rennke, S., and Geuze, H. J . (1997) J ournal of Cell Science 110, 65- 73.

19. Barpeled, M., Conceicao, A. D., Frigerio, L., and Raikhel, N. V. (1995) Plant Cell 7, 667-676. 
20. Roderick, H. L., Campbell, A. K., and Llewellyn, D. H. (1997) FEBS Letters 405, 181- 185.

21. McCauliffe, D. P., Yang, Y. S., Wilson, J ., Sontheimer, R. D., and Capra, J . D. (1992) J ournal of Biological Chemistry 267, 25572562.

22. Sonnichsen, B., Fullekrug, J ., Van, P. N., Diekmann, W., Rob- inson, D. G., and Mieskes, G. (1994) J ournal of Cell Science 107, 2705- 2717.

23. Lodish, H. F., Kong, N., and Wikstrom, L. (1992) J ournal of Biological Chemistry 267, 12753-12760.

24. Beh, C. T., and Rose, M. D. (1995) Proceedings of the National Academy of Sciences of the United States of America 92, 98209823. 